\title{
Essais de production et de collecte d'embryons chez la vache Somba
}

\author{
F. Cristofori ${ }^{1 *}$ G. Quaranta ${ }^{1}$ M. Sidibé ${ }^{2}$ \\ M. Mattoni ${ }^{3}$ G. Trucchi ${ }^{1}$ D. Belemsaga ${ }^{2}$
}

Mots-clés

Bovin Somba - Embryon - Transfert embryonnaire - Biodiversité -

Conservation de la nature -

Bénin - Togo.

\begin{abstract}
Résumé
Des essais de collecte d'embryons ont été menés dans un troupeau de vaches trypanotolérantes de race Somba, pendant quatre cycles de polyovulation réalisés en différentes saisons. Un progestatif (Norgestomet, Crestar ${ }^{\circledR}$ Intervet) et des gonadotrophines (soit p-FSH Pluset ${ }^{\circledR}$ Serono, soit Folltropin ${ }^{\circledR}$ Vetrepharm) ont été utilisés selon des dosages modifiés en raison du poids des animaux. Les donneuses ont été ensuite fécondées deux fois à 12 heures d'intervalle, par saillie naturelle ou insémination artificielle, la semence de trois taureaux ayant été récoltée et congelée sur place en paillettes. Le taux général de réponse à la polyovulation a été de 72 p. 100. Les embryons ont été collectés à 6,5 jours, au stade de morula compactée ou de jeune blastocyste. Sur I'ensemble des 30 collectes, 87 embryons ont pu être récupérés. Parmi ces embryons, 39 (45 p. 100) appartenaient aux catégories Q1 et Q2 et étaient congelables, 19 (22 p. 100) appartenaient à la catégorie Q3 et les 29 restants (33 p. 100) appartenaient à la catégorie Q4 (non transférables). La production moyenne d'embryons transférables (1,9 par donneuse) n'a pas été affectée significativement par le type de gonadotrophine utilisé, mais le taux d'embryons congelables a été plus élevé en saison humide fraîche qu'en saison humide chaude (respectivement 59 vs 38 p. 100).
\end{abstract}

\section{INTRODUCTION}

Dans les régions d'Afrique occidentale et centrale existent des races bovines autochtones de type taurin (Bos taurus) à courtes cornes $(11,14)$. Celles-ci se distinguent des zébus par leur trypanotolérance $(6,26)$. Parmi les différentes races taurines de l'Afrique de l'Ouest, on retrouve les N'Dama, Lagunaire, Muturu, mais également les animaux Somba répartis entre le Togo et le Bénin (25). Cette race bovine se caractérise par son format réduit,

\footnotetext{
1. Dipartimento di Patologia Animale, Università di Torino, Via Leonardo da Vinci, 44 Grugliasco (Torino), Italia

2. Cirdes 559, rue 5-31 angle avenue du Général Louveau, Bobo-Dioulasso, Burkina Faso

3. Cisra, Università di Torino, Via Leonardo da Vinci, 44 Grugliasco (Torino), Italia

* Auteur pour la correspondance

Tél. : +390116709046 ; fax +390116709057 ; e-mail : trucchi@veter.unito.it
}

L'étude a été réalisée dans le cadre Inco-dc (International Cooperation with Developing Countries 1994-1998), contrat Erbic18ct960031 sa robustesse, sa parfaite adaptation au milieu, ainsi que par sa faible sensibilité à la trypanosomose. Le cheptel est actuellement estimé à 25000 têtes (12) mais le métissage avec des zébus afin d'obtenir des animaux d'un plus grand gabarit, l'abattage des mâles pour des cérémonies ou leur castration précoce entraînent une diminution constante de ce cheptel. Le bétail trypanotolérant patrimoine inaliénable de la biodiversité - est considéré comme un des outils pour mettre en valeur les zones de l'Afrique subsaharienne infestées par les glossines $(17,18)$. La conservation de ce cheptel est facilitée par l'utilisation des biotechnologies de la reproduction. Leur application au niveau du continent africain reste cependant problématique, en particulier pour les races taurines autochtones $(7,8)$, à cause de la nécessité d'une adaptation des techniques de stimulation de la polyovulation et de collecte des embryons imposée par les caractéristiques anatomiques et physiologiques particulières de ces races et par les différentes conditions de terrain (5). Ce travail entre dans le cadre des programmes de sauvegarde des races trypanotolérantes soit in situ soit ex situ (4), en mettant au point la technique de production et de collecte d'embryons chez la race taurine Somba. 


\section{MATERIEL ET METHODES}

\section{Les milieux}

L'étude a porté sur des animaux Somba provenant de troupeaux conduits de manière traditionnelle dans la région Ouest africaine de l'Atacora (Bénin). Cette région est comprise entre $9^{\circ} 30^{\prime}$ et $11^{\circ}$ de lat. $\mathrm{N}$, et $0^{\circ} 30^{\prime}$ et $1^{\circ}$ de long. E, et elle est considérée comme le berceau de la race Somba. Par la suite, les animaux ont été transportés au Burkina Faso à la station expérimentale du Cirdes à Banankélédaga, situé à $11^{\circ} 8^{\prime}$ de lat. $\mathrm{N}$ et $4^{\circ} 11^{\prime}$ de long. $\mathrm{O}$, à environ $20 \mathrm{~km}$ au nord de Bobo-Dioulasso. Cette étude a été menée de novembre 1997 à juin 2000. Les milieux de provenance et de destination, classés comme zone subhumide (1), sont similaires, du point de vue du climat. Les températures minimales et maximales varient respectivement entre 17 et $23^{\circ} \mathrm{C}$ et entre 33 et $37^{\circ} \mathrm{C}$; la pluviométrie annuelle moyenne est d'environ $1100 \mathrm{~mm}$. La saison des pluies s'étend de juin à octobre. On y reconnaît quatre saisons différentes : la saison sèche fraîche $(\mathrm{SF})$ de novembre à février, la saison sèche chaude (SC) de mars à mai et la saison des pluies qui est divisée en saison des pluies fraîche (HF) de juin à août et en saison des pluies chaude $(\mathrm{HC})$ de septembre à octobre.

\section{Choix du matériel animal}

Le troupeau dont les auteurs disposaient à l'origine était composé de 24 femelles et de trois mâles choisis selon des critères génétiques et d'aptitude à la reproduction. Ainsi l'identification des animaux dans les exploitations à été faite sur une base morphologique (absence de bosse, fanon réduit, courtes cornes) ; puis, ils ont été testés pour les polymorphismes biochimiques suivants : $\mathrm{Hb}$, Tf, et facteur Z' afin d'établir leur appartenance au type taurin $(14,23,24)$.

La sélection sur les critères de reproduction a tenu compte, vu le manque d'informations sur le passé des animaux, de la présence à la palpation rectale d'un corps jaune et du fait que les femelles avaient déjà vêlé au moins une fois. Les mâles ont été choisis sur la base de leur capacité reproductive évaluée par une enquête rapide sur le terrain, leur morphologie testiculaire et leur docilité.

Tous les sujets, avant d'être retenus, ont été soumis au test du rose bengale pour la brucellose et à la tuberculinisation. Avant le transport et après traitement avec de l'acéturate de diaminazène contre les trypanosomes (Berenil ${ }^{\circledR}$ - Hoechst, $7 \mathrm{mg} / \mathrm{kg}$ ) et avec de la fluméthrine contre les ectoparasites (Bayticol pour-on ${ }^{\circledR}$, Bayer, $1 \mathrm{mg} / \mathrm{kg}$ ), ils ont été vaccinés contre la peste bovine et la pasteurellose.

\section{Conduite à la ferme expérimentale}

A la ferme expérimentale du Cirdes, les animaux (mâles et femelles séparés) ont passé une période d'adaptation d'environ 90 jours. Pendant la saison sèche, ils ont été alimentés avec $3 \mathrm{~kg}$ de paille de riz, plus $300 \mathrm{~g}$ de tourteau de coton et $100 \mathrm{~g}$ de mélasse par animal et par jour. En saison des pluies, ils pâturaient de $8 \mathrm{~h}$ jusqu'à $17 \mathrm{~h}$ sur des parcelles fourragères (association d'une graminée, Panicum maximum cv C1, et d'une légumineuse, Stylosanthes hamata cv Verano); l'eau était offerte ad libitum.

Les trois taureaux ont été dressés à la collecte du sperme au moyen du vagin artificiel et la semence récoltée, après évaluation au laboratoire, a été congelée sur place en paillettes moyennes $(0,5 \mathrm{ml}$, dilution avec Biociphos Plus ${ }^{\circledR}$, Imv). Chaque dose contenait un minimum de huit millions de spermatozoïdes mobiles.

Après l'observation des chaleurs, induites par l'administration de $500 \mu \mathrm{g}$ de cloprosténol (Estrumate ${ }^{\circledR}$, Shering-Plough) et observées selon Mattoni et coll. (15), les vaches donneuses ont été sélectionnées selon les critères proposés par Nibart et Bouyssou (20), ce qui a permis, en février 1998, de retenir un troupeau composé de 16 femelles.

Au total, quatre cycles de polyovulation ont été réalisés, le premier en saison sèche (février 1998, SF, $\mathrm{n}=10$ ), deux en saison humide chaude (septembre 1998, $\mathrm{HC}_{1}, \mathrm{n}=15$; septembre 1999, $\mathrm{HC}_{2}, \mathrm{n}=15$ ) et le dernier en saison humide fraîche (juin 2000, HF, $\mathrm{n}=10$ ).

\section{La polyovulation}

Le protocole de polyovulation a prévu l'utilisation d'un progestatif (implant de $3 \mathrm{mg}$ de Norgestomet, Crestar ${ }^{\circledR}$ Intervet) et de deux différentes gonadotrophines (p-FSH Pluset ${ }^{\circledR}$ Serono : 500 UI FSH 500 UI LH porcine $/ 20 \mathrm{ml}$; Folltropin ${ }^{\circledR}$ Vetrepharm : équivalent de $400 \mathrm{mg}$ NIH-FSH-P1 porcine $/ 20 \mathrm{ml}$ ), selon des protocoles modifiés en raison du poids des animaux, nettement inférieur à celui des animaux sur lesquels les protocoles classiques (22) ont été mis au point. Les donneuses ont été reparties en quatre lots en fonction du poids $(<150 \mathrm{~kg}$ et $>150 \mathrm{~kg}$ ) et de la gonadotrophine utilisée $\left(\mathrm{P}_{1}<150 \mathrm{~kg}\right.$, Pluset ${ }^{\circledR} ; \mathrm{P}_{2}>150 \mathrm{~kg}$, Pluset ${ }^{\circledR} ; \mathrm{F}_{1}<150 \mathrm{~kg}$, Folltropin $^{\circledR}$; et $F_{2}>150 \mathrm{~kg}$, Folltropin ${ }^{\circledR}$ ). Le tableau I résume le schéma des traitements effectués.

Le nombre d'animaux appartenant aux lots mentionnés ainsi que la méthode de polyovulation et d'insémination sont reportés dans le tableau II.

Afin de simplifier l'analyse des données, les deux sessions de polyovulation en $\mathrm{HC}_{1}$ et $\mathrm{HC}_{2}$ ont été traitées comme une seule session dénommée $\mathrm{HC}$.

Les donneuses ayant eu au minimum un nombre de corps jaunes supérieur ou égal à deux ont été considérées comme des réponses positives et les donneuses n'ayant pas ovulé ou ayant eu un nombre de corps jaunes inférieur à deux comme des réponses négatives.

\section{Collecte des embryons}

Les embryons ont été collectés à 6,5 jours, au stade de morula compactée ou de jeune blastocyste (27). La mise au point de la technique de récolte non chirurgicale a été effectuée en SF (février 1998). A cause du gabarit réduit de ces vaches et de la petite taille de leur tractus génital, la palpation transrectale et le placement de la sonde (cathéter de polypropylène : deux voies à diamètre très réduit, Rush CH08) ont nécessité la mise au point des méthodes de collecte originales. L'administration de l'anesthésie épidurale (4-5 $\mathrm{ml}$ de xylocaïne 2 p. 100 selon la taille) afin de permettre le placement de la sonde s'est révélée d'une valeur indéniable pour le succès des lavages utérins. Chez les vaches d'une taille plus importante (> $150 \mathrm{~kg}$ ), la sonde a été placée selon la méthode classique. En revanche, chez les plus petites vaches $(<150 \mathrm{~kg})$ il a fallu recourir à une technique, dite à l'italienne, dérivée de la méthode d'insémination artificielle (IA) : au moyen de pinces d'Allis, le col utérin a été pris et extériorisé au niveau de la vulve, puis, à l'aide de la palpation transvaginale, le cathéter a pu être introduit. Une fois celui-ci positionné correctement, la suite de la collecte a été conduite selon la méthode classique de récolte embryonnaire (19). Chaque corne de l'utérus a été lavée avec $300 \mathrm{ml}$ de solution Pbs (phosphate buffered saline) additionnée de $2 \mathrm{~g} / \mathrm{l}$ d'albumine bovine fraction V. Le liquide récupéré a été filtré avec des filtres Mini Flush in-line DO5A (Minitüb, Floride, Etats-Unis), dont le côté quadrillé est aussi utilisable pour la recherche au microscope stéréoscopique.

Les premières collectes $(n=6)$ faites en saison SF n'ont pas été incluses dans le calcul des embryons récoltés, une panne de la loupe n'ayant pas permis une détermination fiable du nombre d'embryons récoltés. En revanche, les vaches polyovulées durant 
cette séance ont été prises en compte dans le calcul des réponses à la polyovulation.

La recherche des embryons a été effectuée à l'aide d'un microscope stéréoscopique binoculaire à petit agrandissement (x 15). Une fois les embryons identifiés, ils ont été évalués selon les indications de l'Iets $(\mathrm{Q} 1-\mathrm{Q} 2=$ congelables; $\mathrm{Q} 3=$ transférables frais ; $\mathrm{Q} 4$ = non transférables) (27).

Malgré le manque d'homogénéité des populations considérées et la différence numérique des échantillons, les résultats des réponses à la polyovulation et des collectes d'embryons ont été vérifiés par le test du $\chi^{2}$ et soumis à la comparaison de deux pourcentages.

\section{RESULTATS}

\section{Réponse à la polyovulation}

Au total, 50 traitements ont été effectués dont 19 avec Pluset ${ }^{\circledR}$ (37 p. 100) et 31 avec Folltropin ${ }^{\circledR}(63$ p. 100) (tableau II). Les résultats ont montré que 36 traitements $(72$ p. 100) ont donné des

\section{Tableau I}

Schéma récapitulatif des traitements de polyovulation selon le poids des animaux

\begin{tabular}{|c|c|c|c|c|c|}
\hline \multicolumn{2}{|c|}{ Traitement } & \multicolumn{2}{|c|}{ Pluset $^{\circledR}$ (UI) } & \multicolumn{2}{|c|}{ Folltropin $^{\circledR} *$} \\
\hline Jour & Heure & $\begin{array}{c}<150 \mathrm{~kg} \\
\text { Lot } \mathrm{P}_{1}\end{array}$ & $\begin{array}{l}>150 \mathrm{~kg} \\
\text { Lot } P_{2}\end{array}$ & $\begin{array}{c}<150 \mathrm{~kg} \\
\text { Lot } \mathrm{F}_{1}\end{array}$ & $\begin{array}{c}>150 \mathrm{~kg} \\
\text { Lot } \mathrm{F}_{2}\end{array}$ \\
\hline 1 & 8 h 00 & Implant norgestomet & & & \\
\hline 6 & $\begin{array}{r}8 \text { h } 00 \\
20 \text { h } 00\end{array}$ & $\begin{array}{l}\text { FSH } 37,50+\text { LH } 37,50 \\
\text { FSH } 31,25+\text { LH } 31,25\end{array}$ & $\begin{array}{l}\text { FSH } 62,50+\text { LH } 62,50 \\
\text { FSH 50,00 + LH 50,00 }\end{array}$ & $\begin{array}{l}\text { p-FSH } 30 \\
\text { p-FSH } 30\end{array}$ & $\begin{array}{l}\text { p-FSH } 50 \\
\text { p-FSH } 50\end{array}$ \\
\hline 7 & $\begin{array}{r}8 \text { h } 00 \\
20 \text { h } 00\end{array}$ & $\begin{array}{l}\text { FSH } 31,25+\text { LH } 31,25 \\
\text { FSH } 25,00+\text { LH } 25,00\end{array}$ & $\begin{array}{l}\text { FSH } 37,50+\mathrm{LH} 37,50 \\
\text { FSH } 25,00+\mathrm{LH} 25,00\end{array}$ & $\begin{array}{l}\text { p-FSH } 24 \\
\text { p-FSH } 24\end{array}$ & $\begin{array}{l}\text { p-FSH } 40 \\
\text { p-FSH } 40\end{array}$ \\
\hline 8 & $\begin{array}{r}8 \text { h } 00 \\
20 \text { h } 00 \\
20 \text { h } 00\end{array}$ & $\begin{array}{l}\text { FSH } 25,00+\text { LH 25,00 } \\
\text { FSH } 12,50+\text { LH 12,50 }\end{array}$ & $\begin{array}{c}\text { FSH 25,00+ LH 25,00 } \\
\text { FSH 12,50+ LH 12,50 } \\
\operatorname{PgF}_{2 \alpha}\end{array}$ & $\begin{array}{l}\text { p-FSH } 18 \\
\text { p-FSH } 18\end{array}$ & $\begin{array}{l}\text { p-FSH } 30 \\
\text { p-FSH } 30\end{array}$ \\
\hline 9 & $\begin{array}{r}8 \text { h } 00 \\
20 \text { h } 00\end{array}$ & $\begin{array}{l}\text { FSH } 12,50+\text { LH } 12,50 \\
\text { FSH } 12,50+\text { LH 12,50 }\end{array}$ & $\begin{array}{l}\text { FSH } 12,50+\mathrm{LH} 12,50 \\
\text { Retrait de l'implant } \\
\text { FSH } 12,50+\mathrm{LH} 12,50\end{array}$ & $\begin{array}{l}\text { p-FSH } 12 \\
\text { p-FSH } 12\end{array}$ & $\begin{array}{l}\text { p-FSH } 20 \\
\text { p-FSH } 20\end{array}$ \\
\hline 10 & $\begin{array}{r}8 \text { h } 00 \\
20 \text { h } 00\end{array}$ & 1 ère & $\begin{array}{c}\text { CEstrus } \\
\text { mination (artificielle ou sailli }\end{array}$ & & \\
\hline 11 & 8 h 00 & & mination (artificielle ou saillie & & \\
\hline 17 & 8 h 00 & & Collecte d'embryons & & \\
\hline
\end{tabular}

* mg/équivalent NIH-FSH-P1 (FSH porcine)

\section{Tableau II}

Traitement de polyovulation et méthode d'insémination selon les différentes saisons et le poids

\begin{tabular}{|c|c|c|c|c|c|c|c|c|c|}
\hline Saison & $\begin{array}{c}\text { Lot } P_{1} \\
<150 \mathrm{~kg}\end{array}$ & $\begin{array}{c}\text { Lot } P_{2} \\
>150 \mathrm{~kg}\end{array}$ & Total & $\begin{array}{l}\text { Lot } F_{1} \\
<150 \mathrm{~kg}\end{array}$ & $\begin{array}{c}\text { Lot } F_{2} \\
>150 \mathrm{~kg}\end{array}$ & Total & Saillie & IA & Total \\
\hline SF & $\begin{array}{c}(139,6 \pm 5,9) \\
5 \text { sujets }\end{array}$ & $\begin{array}{c}(169,2 \pm 10,1) \\
5 \text { sujets }\end{array}$ & 10 & 0 & 0 & 0 & 10 & 0 & 10 \\
\hline $\mathrm{HF}$ & 0 & $\begin{array}{c}(161,5 \pm 8,5) \\
4 \text { sujets }\end{array}$ & 4 & $\begin{array}{c}(143,4 \pm 4,5) \\
2 \text { sujets }\end{array}$ & $\begin{array}{c}(167,7 \pm 8,2) \\
4 \text { sujets }\end{array}$ & 6 & 2 & 8 & 10 \\
\hline \multirow{2}{*}{$\begin{array}{l}\mathrm{HC}_{1} \\
\mathrm{HC}_{2}\end{array}$} & $\begin{array}{c}138 \\
1 \text { sujet }\end{array}$ & $\begin{array}{c}(160,5 \pm 6.0) \\
4 \text { sujets }\end{array}$ & 5 & $\begin{array}{c}(140,0 \pm 2,0) \\
2 \text { sujets }\end{array}$ & $\begin{array}{c}(161,1 \pm 6,3) \\
8 \text { sujets }\end{array}$ & 10 & 4 & 11 & 15 \\
\hline & & & & $\begin{array}{c}(140,0 \pm 2,8) \\
2 \text { sujets }\end{array}$ & $\begin{array}{c}(167,1 \pm 11,1) \\
13 \text { sujets }\end{array}$ & 15 & 4 & 11 & 15 \\
\hline Total & 6 & 13 & 19 & 6 & 25 & 31 & 20 & 30 & 50 \\
\hline
\end{tabular}

Lot $\mathrm{P}_{1}$ et $\mathrm{P}_{2}=$ Pluset $^{\circledR}$; lot $\mathrm{F}_{1}$ et $\mathrm{F}_{2}=$ Folltropin $^{\circledR}$; entre parenthèses : poids moyen \pm écart-type $(\mathrm{kg})$

$\mathrm{SF}=$ saison sèche fraîche $; \mathrm{HF}=$ saison humide fraîche $; \mathrm{HC}=$ saison humide chaude $; \mathrm{HC}_{1}=$ septembre $1998 ; \mathrm{HC}_{2}=$ septembre 1999 
réponses positives et 14 (28 p. 100) des réponses négatives. Les taux de réponses positives pour chaque type de traitement gonadotrope ont été de 77,5 p. 100 (24/31) et de 63 p. 100 (12/19) respectivement pour Folltropin ${ }^{\circledR}$ et Pluset ${ }^{\circledR}$ et n'ont pas été significativement différents $\left(\chi^{2}=0,58 ;\right.$ d.1. $\left.=1 ; p=0,275\right)$. Les taux de réponses positives ont été de 58 p. $100(7 / 12)$ et de 76 p. 100 (29/38) pour les donneuses dont le poids a été respectivement inférieur ou supérieur à $150 \mathrm{~kg}$, mais cette différence n'a pas été significative $\left(\chi^{2}=0,226 ;\right.$ d.1. $\left.=1 ; p=0,226\right)$. Les taux de réponses aux traitements en fonction de la saison ont montré que 60 p. 100 (6 sur 10) des sujets ont répondu positivement en $\mathrm{SF}$, alors que ces taux sont montés à 70 p. $100(7 / 10)$ et à 77 p. $100(23 / 30)$ pendant les deux saisons $\mathrm{HF}$ et $\mathrm{HC}$.

Comme aucune vache n'a été soumise au traitement avec Folltropin ${ }^{\circledR}$ en saison SF, l'analyse comparative entre traitements à l'intérieur des saisons n'a été possible que pour les saisons humides HF et HC. Les taux de réponses positives ont été de 75 p. 100 (3/4) et de 66 p. $100(4 / 6)$ en saison HF, et de 60 p. 100 (3/5) et 80 p. 100 $(20 / 25)$ en saison HC, respectivement pour les lots Pluset ${ }^{\circledR}$ et Folltropin $^{\circledR}$, mais la différence n'a pas été significative $\left(\chi^{2}=1,409\right.$; d.l. $=1 ; p=0,084)$.

\section{Collecte des embryons}

Sur l'ensemble des 30 collectes effectuées pendant les deux saisons humides, 87 embryons ont été récoltés, soit une moyenne de 2,9 embryons par collecte. Les moyennes d'embryons collectés ont été de 4,1 (29/7) et de 2,5 (58/23) embryons par collecte respectivement pendant les saisons HF et HC. Les donneuses dont le poids a été supérieur à $150 \mathrm{~kg}$ ont donné en moyenne 3 embryons (75/25), contre 2,4 embryons (12/5) par récolte chez les vaches de moins de $150 \mathrm{~kg}$, soit une hausse de 20 p. 100 en faveur des donneuses les plus lourdes.

La production moyenne d'embryons ayant été similaire pour les deux gonadotrophines utilisées, 3 embryons (71/24) contre 2,6 $(16 / 6)$ par collecte, respectivement pour Folltropin ${ }^{\circledR}$ et Pluset ${ }^{\circledR}$, les deux traitements ont été groupés pour l'analyse des résultats. Des 87 embryons récoltés, 39 (45 p. 100) appartenaient à la catégorie Q1 et Q2, 19 (22 p. 100) appartenaient à la catégorie Q3, tandis que les 29 restants (33 p. 100) appartenaient à la catégorie Q4 (tableau III). Par conséquent, la moyenne des embryons de bonne qualité par collecte a été de 1,3.

Quant à l'effet de la saison de collecte sur la qualité des embryons, en saison HF, 59 p. 100 (17/29) des embryons récoltés ont été notés soit Q1 soit Q2, tandis que ce taux a diminué à 38 p. 100 $(22 / 58)$ en saison $\mathrm{HC}\left(\chi^{2}=2,561\right.$, correction de Yates ; d.l. $=1$; $\mathrm{p}=0,0674)$.

En ce qui concerne l'effet de la méthode d'insémination sur la récolte d'embryons (tableau III), au total 39 embryons ont été obtenus des 10 vaches fécondées par saillie naturelle (moyenne 3,9 ) et 48 embryons des 20 vaches fécondées par IA (moyenne $2,4)$. La moyenne des embryons de bonne qualité $(\mathrm{Q} 1+\mathrm{Q} 2)$ obtenus par collecte a été de 1,5 et de 1,2 , respectivement après saillie naturelle et IA. En considérant la saison, ont été collectés en moyenne 2,5 et 2,4 (HF) ou 1,25 et 0,8 (HC) embryons de bonne qualité, respectivement après saillie naturelle et IA (tableau III).

\section{DISCUSSION}

Au cours du présent essai, ont été considérées comme collectables les vaches ayant présenté un nombre de corps jaunes supérieur ou égal à deux. Chez la vache Baoulé, Bianchi et coll. (2) et Blanvillain (3) ont défini comme positives au traitement de polyovulation les réponses ovariennes donnant un nombre de corps jaunes supérieur ou égal à trois. Cependant, étant donné le manque d'informations sur la physiologie de la reproduction de la race Somba, le taux général de réponses $(72 \mathrm{p} .100)$ et le nombre moyen d'embryons par collecte $(2,9)$ ont été relativement satisfaisants et comparables aux résultats rapportés par les auteurs précédents qui, en outre, ont utilisé la gonadotrophine équine chorionique (eCG) pour induire la polyovulation.

Les différences de nombre et de qualité des embryons collectés au cours des différentes saisons par rapport à la gonadotrophine utilisée ne sont pas statistiquement significatives. Toutefois, le traitement par Folltropin ${ }^{\circledR}$ a donné, globalement, plus de réponses positives que le lot traité par Pluset ${ }^{\circledR}(77,5$ vs 63 p. 100) et le nombre

Tableau III

Grille d'évaluation des embryons selon le manuel lets * en fonction de la saison de collecte et de la méthode d'insémination

\begin{tabular}{|c|c|c|c|c|c|c|c|c|c|}
\hline & & \multicolumn{7}{|c|}{ Qualité } & \multirow[b]{2}{*}{ Total } \\
\hline & & \multicolumn{2}{|c|}{ Q1 } & \multicolumn{2}{|c|}{ Q2 } & \multicolumn{2}{|c|}{ Q3 } & Q4 & \\
\hline \multicolumn{2}{|c|}{ Saison de collecte } & M & $\mathrm{BI}$ & M & $\mathrm{BI}$ & M & $\mathrm{BI}$ & $M$ & \\
\hline & 7 & 5 & 2 & 10 & & 4 & & 8 & 29 \\
\hline $\mathrm{HC}$ & 23 & 4 & 3 & 7 & 8 & 12 & 3 & 21 & 58 \\
\hline \multicolumn{2}{|c|}{ Total 30} & 9 & 5 & 17 & 8 & 16 & 3 & 29 & 87 \\
\hline & & \multicolumn{4}{|c|}{39} & \multicolumn{2}{|c|}{19} & 29 & \\
\hline & HF 5 collectes & \multicolumn{4}{|c|}{12} & \multicolumn{2}{|c|}{2} & 6 & 20 \\
\hline & HC 15 collectes & \multicolumn{4}{|c|}{12} & \multicolumn{2}{|c|}{7} & 9 & 28 \\
\hline \multirow[t]{2}{*}{ SN } & HF 2 collectes & \multicolumn{4}{|c|}{5} & \multicolumn{2}{|c|}{2} & 2 & 9 \\
\hline & HC 8 collectes & \multicolumn{4}{|c|}{10} & \multicolumn{2}{|c|}{8} & 12 & 30 \\
\hline
\end{tabular}

* Stringfellow D.A., Seidel S.M., 1998, Iets

$\mathrm{M}=$ morula $; \mathrm{Bl}=$ blastocyste $; \mathrm{IA}=$ insémination artificielle $; \mathrm{SN}=$ saillie naturelle 
d'embryons de bonne qualité a été supérieur dans les lots traités avec Folltropin ${ }^{\circledR}$ (34/24 soit 1,4 embryon par lavage) que dans ceux qui ont été traités avec Pluset ${ }^{\circledR}$ (5/6 soit 0,8 embryon par lavage). Cela pourrait s'expliquer par un rapport FSH : LH plus favorable dans le cas de Folltropin ${ }^{\circledR}$ où seulement des traces de LH ont été présentes, ce qui n'a pas été le cas de Pluset ${ }^{\circledR}$ où le rapport $\mathrm{FSH} / \mathrm{LH}=1$ (tableau I). Un excès comme une insuffisance de LH diminuent la stimulation ovarienne, en particulier, des niveaux élevés de $\mathrm{LH}$ peuvent réduire la réponse à la polyovulation chez la vache $(9,16)$.

Le très faible taux de réponses positives en saison SF pourrait s'expliquer par deux facteurs : la plus modeste réponse au Pluset ${ }^{\circledR}$ et la saison défavorable, facteurs aggravés par le fait que les animaux n'ont pas bénéficié d'un temps suffisamment long pour s'adapter aux nouvelles conditions.

Comme il n'était pas possible de compter sur une distribution homogène du nombre de collectes à l'intérieur des lots et surtout sur un effectif suffisamment important, l'effet saison sur la qualité des embryons n'a pas pu être clairement mis en évidence, bien que la valeur trouvée pour le $\chi^{2}$ ait été proche du niveau de signification. En effet, en saison HF le taux d'embryons de bonne qualité a été plus élevé (58 vs 38 p. 100) qu'en saison HC ; cela pouvait être dû à l'effet négatif des températures élevées sur les premiers stades du développement embryonnaire (10).

Si l'on considère le nombre d'embryons obtenus par collecte, il ressort non seulement que les moyennes les plus élevées ont été obtenues en saison HF, mais aussi que dans cette saison il a été récolté un nombre plus élevé d'embryons de bonne qualité par donneuse quelle qu'ait été la méthode de fécondation utilisée.

\section{BIBLIOGRAPHIE}

1. Assessment of Animal Agriculture in Sub-Saharan Africa, 1992. Morrilton, AR, USA, Winrock International Institute for Agricultural Development, p. 14-18.

2. BIANCHI M., CHICOTEAU P., CLOE C., BASSINGA A., 1986. Premiers essais de transferts d'embryons sur bovins de race Baoulé au Burkina Faso. Revue Elev. Méd. vét. Pays trop., 39 : 139-144.

3. BLANVILLAIN C., 1993. Expérience du Crta dans le domaine du transfert d'embryons. Amélioration génétique des bovins de l'Afrique de I'Ouest. Rome, Italie, Fao, p. 199-203. (Etudes Production et santé animale $\left.n^{\circ} 110\right)$

4. BREM G., BRENIG B., MULLER M., SPRINGMANN K., 1989. Ex situ Cryoconservation of genomes and genes of endangered cattle breeds by means of modern biotechnological methods. Rome, Italy, FAO, $123 \mathrm{p}$. (Animal Production and Health Paper No 76)

5. CALLESEN H., GREVE T., 1996. Superovulation of cattle. In: Proc. 12th Meeting AETE, Lyon, France, 13-14 September 1996, p. 85-87.

6. CARR W.R., MACLEOD J., WOOLF B., SPOONER R.L., 1974. A survey of the relationship of genetic markers, tick infestation level and parasitic diseases in zebu cattle in Zambia. Trop. Anim. Health Prod., 6: 203-214.

7. CHICOTEAU P., 1987. Perspectives et réalité du transfert d'embryons en Afrique. In : Proc. International Embryo Movement Symposium, Montréal, Canada, 1987-1988, p. 41-53, 43 ref.

8. CHICOTEAU P., 1991. La reproduction des bovins tropicaux. Rec. Méd. vét., $167: 241-247$.

\section{CONCLUSION}

Le but de l'étude a été de vérifier la possibilité d'application chez la vache Somba des traitements de polyovulation en vue du transfert embryonnaire. Bien que le gabarit des sujets traités ait été très réduit, cela n'a pas constitué un facteur limitant pour la collecte non chirurgicale d'embryons, compte tenu des modifications apportées à la méthode classique.

Les conclusions de Bianchi et coll. (2) sur la réponse ovarienne à eCG des bovins trypanotolérants ont été confirmées, après l'emploi de FSH, avec des réponses qui se sont révélées suffisamment fiables et reproductibles. Cependant, la réponse ovarienne au traitement gonadotrope a été réduite par rapport à celle des races européennes (5,5 embryons transférables par donneuse) (13).

Il ne semble pas que la saison ait exercé un rôle important sur la réponse ovarienne mais les températures plus basses semblent avoir eu un effet positif sur la quantité et sur la qualité des embryons. Toutefois, la réalisation des essais en milieu contrôlé serait plus propice à leur succès, compte tenu des meilleures conditions d'alimentation et d'entretien $(7,21,28)$.

Le taux élevé d'embryons de mauvaise qualité pourrait s'expliquer par la faible fertilité des taureaux utilisés pour la collecte de la semence destinée à l'IA aussi bien que pour les saillies naturelles ; cet aspect nécessite donc des études ultérieures.

Les méthodes utilisées n'ont pas été tout à fait exemptes de contraintes mais leur réussite a été tributaire de l'appui des organismes locaux. A l'heure actuelle auprès du Cirdes une petite banque de semences et d'embryons de cette race a été installée afin de contribuer à la conservation de la biodiversité.
9. CHUPIN D., COMBARNOUS Y., PROCUREUR R., 1984. Antagonistic effect of $\mathrm{LH}$ on FSH-induced superovulation in cattle. Theriogenology, 21: 229.

10. EDWARDS J.L., EALY A.D., MONTERROSO V.H., HANSEN P.J., 1997. Ontogeny of temperature-regulated heat shock protein 70 synthesis in preimplantation bovine embryos. Mol. Reprod. Dev., 48: 25-33.

11. EPSTEIN H., 1971. The origin of the domestic animals of Africa, Vol. 1. New York, NY, USA, Pub. Africana, p. 573.

12. HALL S.J.G., GNAHO L.K., MEGHEN C., 1995. Une enquête sur la race bovine Somba au Bénin. Revue Elev. Méd. vét. Pays trop., $48: 77-83$.

13. HEYMAN Y., 2001. Overall bovine embryo transfer activity in Europe in 2000. In: Proc. AETE, Lyon, France, 7-8 September 2001, 17, p. 61.

14. MAILLARD J.C., CONGO I., BASSINGA A., CUVEILLIER J.F., 1992 Immunogénétique du taurin Baoulé en pays Lobi (Burkina Faso). I. Environnement de cette population trypanotolérante. Revue Elev. Méd. vét. Pays trop., $45:$ 63-68.

15. MATTONI M., MUKASA-MURGERWA E., CECCHINI G., SOVANI S., 1988. The reproductive performance of East African (Bos indicus) zebu cattle in Ethiopia. 1. Estrus cycle length, duration, behavior and ovulation time. Theriogenology, 30: 961-971.

16. MURPHY B.D., MAPLETOFT R.J., MANNS J., HUMPHREY W.D., 1984. Variability in gonadotrophin preparations as a factor in the superovulatory response. Theriogenology, 21: 117-125.

17. MURRAY M., BLACK S.J., 1985. African trypanosomiasis in cattle: working with nature's solution. Vet. Parasitol., 18: 167-82. 
18. MURRAY M., TRAIL J.C., D'IETEREN G.D., 1990. Trypanotolerance in cattle and prospects for the control of trypanosomiasis by selective breeding. Revue sci. tech., 9: 369-86.

19. NEWCOMB R., 1982. Egg recovery and transfer in cattle. In: Adams C.E. Ed., Mammalian egg transfer. Boca Raton, FL, USA, CRC Press, p. 81-118.

20. NIBART M., BOUYSSOU B., 1981. Le transfert embryonnaire chez les bovins. Rec. Méd. vét., 157 : 71-87.

21. NIIE A., AGYEMANG K., 1991. Performance of a station-managed N'Dama herd in the Gambia. Trop. Anim. Health Prod., 23: 45-54.

22. PONZIO P., QUARANTA G., VINCENTI L., 1992. Correlazione fra qualità embrionale e livelli ormonali in corso di superovulazione nella bovina piemontese. In: $4^{\circ}$ Meeting nazionale Studio dell'efficienza riproduttiva degli animali di interesse zootecnico, Bergamo, Italia, 10 aprile 1992, p. 115-119.

23. QUEVAL R., 1982. Polymorphisme de la transferrine chez les bovins trypanosensibles et trypanotolérants de l'Afrique de l'Ouest. Répartition et fréquence de leurs allèles. Revue Elev. Méd. vét. Pays trop., 35 : 373-380.
24. QUEVAL R., PETIT J.P., 1982. Polymorphisme biochimique de I'hémoglobine des populations bovines trypanosensibles, trypanotolérantes et de leur croisements dans I'Ouest africain. Revue Elev. Méd. vét. Pays trop., 35 : 137-146.

25. REGE J.E.O., ABOAGYE G.S., TAWAH C.L., 1994. Shorthorn cattle of West and Central Africa. I. Origin, distribution, classification and population statistics. World Anim. Rev., 78: 2-48.

26. SAUVEROCHE B., WAGNER H.G., 1993. Physiologie de la reproduction des bovins trypanotolérants. Rome, Italie, Fao, p. 199-203. (Etudes Production et santé animale $\mathrm{n}^{\circ} 112$ )

27. STRINGFELLOW D.A., SEIDEL S.M., 1998. Manual of IETS, 3rd Edn. Savoy, IL, USA, International Embryo Transfer Society, p. 106-107.

28. YOUSSAO A.K.I., AHISSOU A., TOURE Z., LEROY P.L., 2000. Productivité de la race Borgou à la Ferme d'élevage de l'Okpara au Bénin. Revue Elev. Méd. vét. Pays trop., 53 : 67-74.

Reçu le 10.07.2001, accepté le 06.06.2002

\section{Summary}

Cristofori F., Quaranta G., Sidibé M., Mattoni M., Trucchi G., Belemsaga D. Preliminary Trials on Embryo Production and Collection in the Somba Cow

Trials were conducted to collect embryos in a herd of trypanotolerant Somba cows. The trial period covered four superovulation cycles induced during various seasons. A progestagen (Norgestomet, Crestar ${ }^{\circledR}$ Intervet) and gonadotropins (p-FSH Pluset ${ }^{\circledR}$ Serono, or Folltropin ${ }^{\circledR}$ Vetrepharm) were used at various doses according to the animal weight. The donors were then fertilized twice at 12-hour interval, either by natural or artificial insemination, the semen of three bulls having been previously collected and frozen in straw on the site. The overall superovulation response rate was $72 \%$. The embryos were collected at 6.5 days at the stage of compacted morula or young blastocyst. Of the 30 flushings performed, 87 embryos were recovered. Among them, 39 (45\%) belonged to categories Q1 and Q2, and could be cryopreserved, $19(22 \%)$ belonged to category Q3, and the remaining 29 (33\%) belonged to category Q4 (non-viable). The average production of viable embryos (1.9 per donor) was not significantly affected by the type of gonadotropin used. However, the rate of embryos that could be selected for cryopreservation was higher in the cool rainy season than in the hot rainy season (59 vs 38\%, respectively).

Key words: Somba cattle - Animal embryo - Embryo transfer - Biodiversity - Nature conservation - Benin - Togo.

\section{Resumen}

Cristofori F., Quaranta G., Sidibé M., Mattoni M., Trucchi G., Belemsaga D. Ensayos en la producción y la recolecta de embriones en la vaca Somba

Se llevaron a cabo ensayos de recolecta de embriones en un hato de vacas tripanotolerantes de la raza Somba, durante cuatro ciclos de poli ovulación, llevados a cabo en diferentes estaciones. Se utilizó un progestageno (Norgestomet, Crestar ${ }^{\circledR}$ Intervet) y gonadotropinas (ya sea p-FSH Pluset ${ }^{\circledR}$ Serono, o Folltropin ${ }^{\circledR}$ Vetrepharm) según dosis modificadas de acuerdo al peso de los animales. Las donadoras fueron luego fecundadas dos veces, con 12 horas de intervalo, por salto natural o inseminación artificial, utilizando el semen de tres toros recolectado y congelado in situ en pajillas. La tasa general de respuesta a la poli ovulación fue de $72 \%$. Los embriones se recolectaron a 6,5 días, en el estadio de mórula compacta o de blastocisto joven. Sobre un total de 30 colectas, se recuperaron 87 embriones. Entre estos embriones, 39 (45\%) pertenecieron a las categorías Q1 y Q2 y fueron congelables, 19 $(22 \%)$ pertenecieron a la categoría Q3 y los 29 (33\%) restantes pertenecieron a la categoría Q4 (no transferibles). La producción promedio de embriones transferibles (1,9 por donadora) no fue afectada significativamente por el tipo de gonadotropina utilizada, pero la tasa de embriones congelables fue más alta durante la estación húmeda fresca que durante la estación húmeda caliente (respectivamente 59 vs $38 \%)$.

Palabras clave: Ganado bovino Somba - Embrión - Transferencia de embriones - Biodiversidad - Conservación de la naturaleza - Benin - Togo. 